\title{
Efectos del cambio climático sobre la producción de aceituna en Yarada, Región Tacna
}

\author{
Effects of climate change on the production of olive in Yarada, Tacna Region
}

Juan Tonconi Quispe ${ }^{1 *}$

\begin{abstract}
RESUMEN
En este artículo se cuantifican los efectos del cambio climático sobre la producción de aceituna en Yarada, Región Tacna, Perú, a partir de las estimaciones del rendimiento de la producción de aceituna mediante el análisis de cointegración y el modelo de vector de corrección de errores (VEC), usando datos de información de 1993-2012. Los resultados del modelo econométrico indican las variables climáticas cómo la temperatura máxima y mínima, precipitación y humedad relativa del suelo afectan significativamente en el rendimiento de la producción de aceituna; la bondad de ajuste o validez del modelo está explicada por más del $70 \%$. En la actualidad la temperatura máxima promedio aún no ha alcanzado el umbral de quiebre $\left(24,07{ }^{\circ} \mathrm{C}\right)$, por lo que aumentos de temperatura mejora el rendimiento de este cultivo; sucede lo contrario con el nivel de temperatura mínima, esta ha sobrepasado el umbral $\left(16,24{ }^{\circ} \mathrm{C}\right)$, caídas de la temperatura mínima tienen efectos desfavorables sobre la productividad de aceituna. Al 2030 se experimentarán descensos leves del rendimiento de la producción de aceituna de 4,94\% en el nivel bajo de pronóstico y $6,42 \%$ en el nivel alto.
\end{abstract}

Palabras clave: aceituna, adaptabilidad, cambio climático, producción agraria.

\begin{abstract}
This paper quantifies the effects of climate change on the production of olive in Yarada, Tacna-Peru region, from yield estimates of the production of olive through cointegration analysis and model of vector error correction (VEC), using 1993-2012 data information. Econometric Model results indicate climatic variables such as maximum and minimum temperature, precipitation and soil humidity significantly affect the performance of the olive harvest, the goodness of fit or validity of the model is explained by more than $70 \%$. At present, the average maximum temperature has not reached the threshold of break $\left(24.07{ }^{\circ} \mathrm{C}\right)$, so that increases in temperature improves the performance of the crop, the opposite happens with the minimum temperature level, this has surpassed the threshold $\left(16.24{ }^{\circ} \mathrm{C}\right)$, the minimum temperature falls has an adverse impact on the productivity of olive. By 2030 it will experience a slight performance drops olive production of $4.94 \%$ in the low and $6.42 \%$ forecast in the high level.
\end{abstract}

Key words: adaptability, agricultural production, climate change, olive.

\section{Introducción}

El cambio climático a nivel regional, nacional e internacional ha causado preocupación entre los científicos y la sociedad civil, en vistas que las variables climáticas como la temperatura, precipitación, nivel del mar, entre otros, medidas del cambio de clima, están siendo severamente afectadas y estas a su vez generan impactos sobre las diversos sectores productivos como el sector agrario, los que se ven afectados en sus rendimientos, aunque los efectos de cambios en el clima sobre la producción de cultivos varía de un país a otro; por lo tanto, los países a partir de la primera Convención Marco de las Naciones Unidas sobre Cambio Climático (CMNUCC), realizada por la ONU (1992) en Rio de Janeiro, Brasil, acuerdan reducir y estabilizar la concentración de Gases de Efecto Invernadero (GEI) causantes del calentamiento global y cambio climático.

Perú, al igual que las naciones vecinas, es uno de los países que será inmensamente afectado por el cambio climático por su ubicación geográfica y por ser una región en desarrollo; primero porque se encuentra cerca de la línea ecuatorial; si las temperaturas de estos territorios ya son elevadas aun sin un severo cambio climático, la actividad agrícola sería insostenible con temperaturas mayores

\footnotetext{
$1 \quad$ Facultad de Ciencias Agropecuarias, Universidad Nacional Jorge Basadre Grohmann, Ciudad Universitaria, Av. Miraflores S/N. Tacna, Perú.

* Autor por correspondencia: ecotonconi@unjbg.edu.pe
} 
a las actuales, lo que afectaría el umbral térmico de las plantas, creando problemas en los cultivos, $\mathrm{y}$ segundo, como indica Cline (2007), para el decenio de 2080 los efectos serían más severos en los países de América Latina, debido a la limitada capacidad de mitigación y adaptabilidad al cambio climático, donde la productividad agrícola se reduce en $24 \%$ sin fertilización carbónica y una reducción del $3 \%$ con fertilización carbónica. Las pérdidas podrían ser devastadoras en algunos de los países más pobres como Senegal y Sudán, las pérdidas en la productividad agrícola superarían el 50\%.

Por su parte, Seo y Mendelsohn (2007) analizan efectos del cambio climático en la agricultura para los países sudafricanos. En general se ha encontrado que la magnitud de los impactos es diferente entre los países, e incluso entre regiones de un mismo país. $\mathrm{Al}$ respecto, basados en una muestra mayor a 2.000 observaciones, estiman que, en promedio, productores grandes y pequeños perderán hasta el $25 \%$ del valor de su flujo de ingresos para 2060. El porcentaje se incrementa hasta $50 \%$ en el escenario climático más severo correspondiente a 2100. Corroborándose a ello Ordaz et al. (2009), El Salvador sufrirá grandes pérdidas en la agricultura ante los efectos climáticos. Para el 2100 el aumento de la temperatura estará entre $2{ }^{\circ} \mathrm{C}$ y $5{ }^{\circ} \mathrm{C}$ y la precipitación aumentará entre $18 \%$ y $40 \%$, lo que traerá una reducción entre $2 \%$ y $8 \%$ del PIB comparado al PIB del 2007.

En la región de Tacna, el cambio climático medido por temperaturas extremas máxima y mínima afecta directamente en la producción agrícola, generando impactos sobre las diversas actividades económicas como la agricultura en distintas zonas de la región, por lo que se constituye en un problema por resolver. El comportamiento de las temperaturas extremas máximas y mínimas en Yarada, durante el período $1993-2012$, han variado en $1{ }^{\circ} \mathrm{C}$ y $1,3{ }^{\circ} \mathrm{C}$, respectivamente (Figura 1); estas variabilidades climáticas han provocado aparición e incremento de las plagas y enfermedades en los cultivos agrícolas en la región, como aceituna, estancando de esta manera la productividad o rendimiento de la producción de aceituna, alcanzando apenas un crecimiento promedio anual de 1,8\%, muy inferior a otros países como España.

El objetivo general de la presente investigación es analizar los efectos del cambio climático sobre la producción de aceituna en Yarada, Región de Tacna, Perú. De forma específica se trata de demostrar que si el cambio de las temperaturas extremas máximas y mínimas, los cambios en los niveles de precipitación y de humedad relativa del suelo, influyen sobre los niveles de productividad de aceituna, y por otro lado se trata de proyectar para el futuro (2030) el comportamiento de los efectos en los niveles de rendimiento de la producción de aceituna.

\section{Materiales y Métodos}

\section{Materiales}

En el presente estudio se utilizan datos de información anual comprendido entre el período de 1993 a 2012, sobre variables relacionadas a la medición del cambio climático como las temperaturas promedio, máximas y mínimas expresadas en grados Celsius $\left({ }^{\circ} \mathrm{C}\right)$, precipitación medida en milímetros $(\mathrm{mm} / \mathrm{mo})$, humedad relativa del suelo en porcentajes (\%), esta información corresponde a la estación

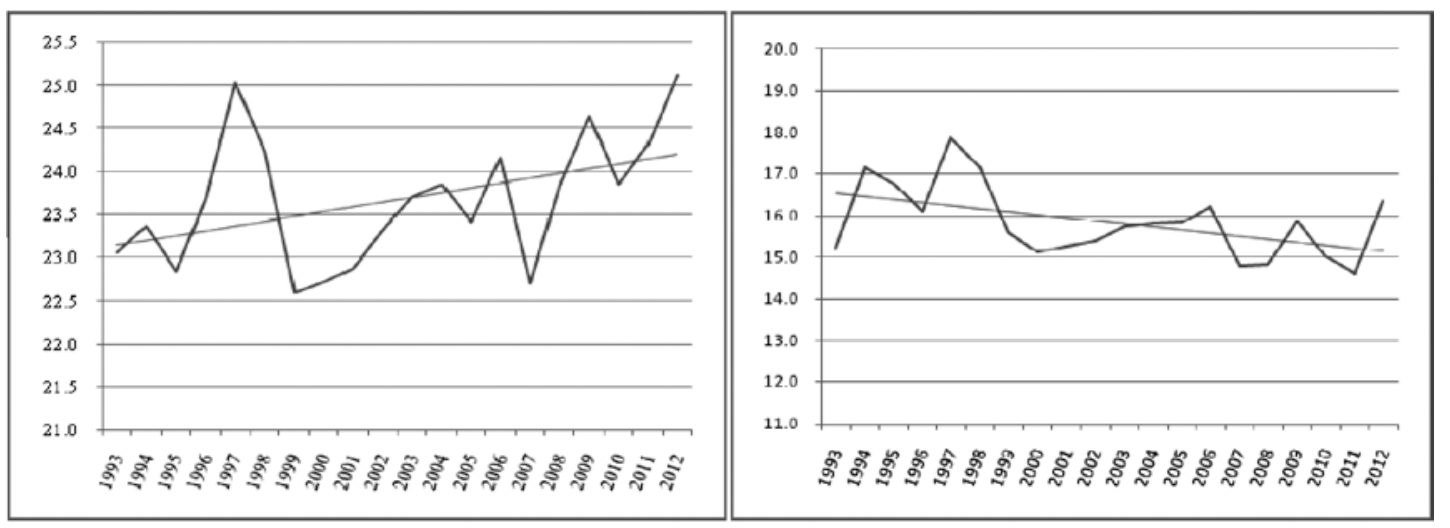

Figura 1. Evolución de temperaturas máximas y mínimas en Yarada-Tacna, período: 1993-2012.

Fuente: SENAMHI (2013). 
meteorológica e hidrológica de Yarada en la Región de Tacna, proporcionado por SENAMHI-Tacna (2013). Mientras las series sobre rendimiento de los producción de aceituna $(\mathrm{kg} / \mathrm{ha})$, participación del sector agropecuario en el producto bruto interno regional y la población de Tacna, obtenida de la Dirección Regional Agraria Tacna (DRAT), Banco Central de Reserva del Perú, filial Arequipa-Tacna (BCRA) e Instituto Nacional de Estadística e Informática Tacna (INEI).

\section{Metodología}

El tipo de investigación es descriptivo, prescriptivo y analítico porque caracteriza, describe, analiza y evalúa los efectos del cambio climático sobre la producción de aceituna en Yarada, para tal efecto se caracteriza la producción de aceituna relacionada con los factores medioambientales, productivos y socioeconómicas, que permitan evaluar y proyectar al futuro los efectos del cambio climático en el rendimiento de la producción de aceituna.

El diseño particular de este tipo de investigación está identificado dentro de las posibilidades que brinda el método científico, mediante el análisis deductivo e inductivo, basado en el procedimiento de la función de producción agrícola.

\section{Modelo de la función de producción}

Una función de producción agrícola relaciona la producción $(\mathrm{Q})$ con variables productivas endógenas (P) como trabajo, capital y otros insumos; con variables exógenos (A) que comprenden variables climáticas e irrigación; y con las características socioeconómicas de los agricultores (S), entre las que se incluyen variables de capital humano y aspectos sociales del productor, según Fleischer et al. (2007).

En términos formales la función de producción agrícola se representa como sigue:

$$
Q_{i}=f\left(P_{i}, S_{i}, A_{i}\right)
$$

Donde $Q_{i}$ puede representar la producción total en el sector agropecuario, la producción en un subsector como por ejemplo el agrícola o el rendimiento por hectárea de un cultivo determinado.

Con fines empíricos, en la región de Tacna la producción de aceituna en el sector agropecuario depende de los factores mencionados anteriormente, por lo que la forma funcional en relación con la teoría económica es la siguiente.

$$
\text { Aceituna }_{i}=f\left(P_{i}, S_{i}, A_{i}\right)
$$

Así, el modelo sirve para analizar un cultivo específico en el tiempo. Es necesario considerar que son funciones de producción agrícola, en principio funciones cuadráticas, raíz cuadrada, tres medios, logarítmicas, translogarítmicas $\mathrm{u}$ otras formas exponenciales como señala Seo y Mendelsohn (2007), que al considerar solo las variables climáticas, con el fin de poder identificar los niveles de temperatura y precipitación que tienen efectos positivos o negativos sobre la producción. Por lo tanto, su forma funcional cuadrática se expresa como:

$$
Q_{i t}=\beta_{0}+\beta_{1} \text { Tem }+\beta_{2} \text { Tem }^{2}
$$

Donde la producción (Qit) depende de la temperatura (Tem) en forma cuadrática, cual permite el análisis de los efectos del cambio climático en el rendimiento de la producción de aceituna, por las estimaciones variables observadas en el tiempo, las que captan la variabilidad del clima, en las temperaturas y precipitaciones, paralelamente se identifican los umbrales de variables climáticas de máximos y mínimos de la temperatura.

El método de estimación que se emplea para alcanzar el objetivo del estudio es por medio del modelo de máxima verosimilitud (MV), mediante Cointegración Johansen o Vector de Corrección de Errores (VEC), con el cual se analizan efectos del cambio climático sobre la productividad de aceituna en Yarada, usando datos de información de series temporales comprendida entre el período 19932012, de esta manera se busca un modelo que sea robusto metodológicamente para explicar cómo las variables climáticas se relacionan con las variables de interés, para después hacer predicciones de cómo evolucionaría la producción en los siguientes años dados distintos escenarios climáticos.

\section{Resultados y Discusión}

\section{Resultados}

El procedimiento utilizado para la estimación y evaluación de los efectos del cambio climático 
en la producción de aceituna es por la metodología de cointegración propuesta por Johansen (1995), que captura las relaciones de equilibrio de largo plazo entre las variables, que permite conocer el comportamiento correlacional, si los resultados esperados se ajustan a lo real y de acuerdo con la teoría económica ambiental (signo esperado). Un primer requisito es que las series sean estacionarias, esto con la finalidad de identificar los vectores de cointegración, y así proceder a estimar relaciones de equilibrio de largo plazo en los modelos multivariados; estas estimaciones requieren seguir el siguiente procedimiento:

a) Orden de integración de las variables

Para determinar el grado de integrabilidad primero se evalúa la presencia de raíz unitaria en las variables utilizando los test de DickeyFuller Aumentado (ADF), Phillips Perron (PP) y Kwiatkowski-Phillips-Schmidt-Shin (KPSS); los resultados de estos test de raíz unitaria se presentan en la Tabla 1, donde nos permite concluir que a un nivel de significancia de $95 \%$ algunas series de las variables tienen problemas de raíz unitaria (son no estacionarías) en niveles como la producción, temperatura máxima, temperatura mínima, precipitación, humedad y la participación porcentual del sector agropecuario en el PIB regional, como se muestra a continuación:
Por otro lado, en las estimaciones realizadas por los test en primeras diferencias indican que todas las series son estacionarias, lo que indica que todas las series son integradas de orden 1. En consecuencia, es factible encontrar relaciones de equilibrio de largo plazo entre las variables mediante la estimación de vectores de cointegración, debido a que las series de las variables incluidas en el modelo tienen el mismo orden de integración.

b) Estimación del modelo de los efectos del cambio climático en la producción de aceituna

En la Tabla 2 se reportan 5 modelos de estimaciones para el cultivo de olivo. Las dos primeras estimaciones relacionan las variables de las temperaturas mínimas y máximas por separado, luego la estimación conjunta de ambas temperaturas y además de considerar variables como la humedad relativa del suelo y la participación porcentual del sector agrícola en el PBI regional, muestran una adecuada bondad de ajuste y los coeficientes son significativos estadísticamente; es decir, que las variables climáticas influyen sobre la productividad de aceituna.

La tercera y cuarta estimación consideran las variables de temperatura mínima y máxima, a cada uno complementa con variables como humedad relativa del suelo y participación porcentual del sector agrario en el PIB regional, muestra que el ajuste de

Tabla 1. Test de raíz unitaria (variables en niveles).

\begin{tabular}{|c|c|c|c|c|c|c|c|}
\hline \multirow[b]{2}{*}{ Variables } & \multicolumn{2}{|c|}{ Dickey Fuller Aumentado 1/ } & \multirow{2}{*}{$\begin{array}{c}\text { AR } \\
(\text { Rezagos })\end{array}$} & \multicolumn{2}{|c|}{ Phillips Perrón 1/ } & \multicolumn{2}{|c|}{ KPSS 2/ } \\
\hline & Constante & $\begin{array}{c}\text { Constante y } \\
\text { tendencia }\end{array}$ & & Constante & $\begin{array}{c}\text { Constante y } \\
\text { tendencia }\end{array}$ & Constante & $\begin{array}{c}\text { Constante y } \\
\text { tendencia }\end{array}$ \\
\hline PR & 0,0683 & $-5,4964$ & 1 & $-2,4029$ & $-5,8807$ & 0,6963 & 0,1384 \\
\hline COSE & 3,3746 & 0,2411 & 0 & 3,3746 & $-0,0792$ & 0,5945 & 0,1832 \\
\hline REN & $-6,3353$ & $-6,5485$ & 0 & $-6,6603$ & $-9,9227$ & 0,2849 & 0,3573 \\
\hline PRE & $-3,5500$ & $-3,4713$ & 0 & $-3,5025$ & $-3,4115$ & 0,0717 & 0,0725 \\
\hline TPRO & $-3,4689$ & $-3,7196$ & 0 & $-3,2206$ & $-3,5919$ & 0,3327 & 0,1875 \\
\hline TMAX & $-2,4853$ & $-2,8561$ & 1 & $-2,3509$ & $-2,6279$ & 0,4211 & 0,0923 \\
\hline TMIN & $-2,5086$ & $-3,1370$ & 1 & $-2,8479$ & $-3,8903$ & 0,3860 & 0,0764 \\
\hline PREC & $-1,0903$ & $-7,9377$ & 1 & $-5,4749$ & $-8,9599$ & 0,1936 & 0,0882 \\
\hline HUM & $-1,7025$ & $-2,6673$ & 1 & $-1,8740$ & $-2,7588$ & 0,4451 & 0,1068 \\
\hline PAGPBI & 0,7129 & $-5,8647$ & 1 & $-0,8816$ & $-5,8647$ & 0,5231 & 0,1454 \\
\hline POB & $-1,6966$ & $-2,4741$ & 1 & 1,5919 & $-2,9344$ & 0,6151 & 0,1258 \\
\hline Valores críticos al $95 \%$ & $-3,0656$ & $-3,673616$ & & $-3,02997$ & $-3,673616$ & 0,463 & 0,146 \\
\hline Valores críticos al $90 \%$ & $-2,6735$ & $-3,277364$ & & $-2,655194$ & $-3,277364$ & 0,347 & 0,119 \\
\hline
\end{tabular}

1/ Ho: La serie es no estacionaria (algunos valores de las variables no son inferiores a los valores críticos (95\% y $90 \%$ ). Por lo tanto no se rechaza $\mathrm{Ho}$ ).

2/ Ho: La serie es estacionaria (algunos valores de las variables no son inferiores a los valores críticos (95\% y $90 \%)$. Por lo tanto se rechaza Ho).

Fuente: Elaboración basada en los datos de información obtenida de SENAMHI-Tacna, Dirección Regional Agraria Tacna, BCRP, INEI. 
Tabla 2. Resultado de las estimaciones en el rendimiento de aceituna.

\begin{tabular}{lccccc}
\hline \multicolumn{1}{c}{ Variables } & Estimación 01 & Estimación 02 & Estimación 03 & Estimación 04 & Estimación 05 \\
\hline Constante & -615070 & -5228619 & 1660935 & -858084 & 902139 \\
TMAX & 51687,07 & & 141262,90 & & $-122219,60$ \\
& {$[1,28]$} & & {$[2,86]^{*}$} & & {$[-2,53]^{*}$} \\
TMAX^2 & $-1073,75$ & $-2964,21$ & & 2598,99 \\
& {$[-1,26]$} & & {$[-2,86]^{*}$} & & {$[2,54]^{*}$} \\
TMIN & & & & 110959 & 67292,92 \\
& & 65404,02 & & {$[22,55]^{*}$} & {$[3,39]^{*}$} \\
TMIN^2 & {$[3,93]^{*}$} & $-2014,06$ & & $-3469,19$ & $-2098,64$ \\
& & {$[-3,92]^{*}$} & & {$[-22,84]^{*}$} & {$[-3,38]^{*}$} \\
HUM & & & 123,23 & $-91,36$ \\
& & & {$[7,52,57$} & {$[6,62]^{*}$} & {$[-1,04]$} \\
PAGPBI & & $-1206,46$ & $-3485,60$ & $-330,30$ \\
R^2 & & {$[-0,85]$} & {$[-5,78]^{*}$} & {$[-0,13]$} \\
R ajustado & 0,79 & 0,88 & 0,77 & 0,54 \\
Log likelihood & 0,73 & 0,85 & 0,54 & 0,64 & 0,32 \\
AK & $-194,50$ & $-197,25$ & $-206,04$ & $-189,42$ & $-164,92$ \\
SCH & 23,61 & 23,92 & 27,34 & 25,49 & 28,10 \\
& 24,50 & 24,81 & 29,32 & 27,50 & 28,44 \\
\hline
\end{tabular}

(*) Estimadores significativos estadísticamente al 5\%.

(**) Estimadores significativos estadísticamente al $10 \%$.

la estimación es adecuado y los coeficientes de los estimadores también son significativos, mostrando un efecto positivo en la producción de aceituna por las variables complementarias.

Para la selección de los mejores modelos se ha utilizado los criterios de $\mathrm{AK}$ y $\mathrm{SCH}$, que explican el comportamiento de los residuales, las mejores estimaciones son 01 y 02 para explicar los efectos de las temperaturas máximas y mínimas en la producción de aceituna en Yarada, Tacna. Asimismo, la bondad de ajuste del modelo (R squared y Log likelihood) indican que estas estimaciones es el adecuado estadísticamente.

Mientras el signo de las variables de temperatura máxima y mínima, humedad relativa del suelo y la participación porcentual del sector agrario en el PIB regional son las esperadas en todos las estimaciones; por lo tanto el análisis de los efectos del cambio climático en la producción de aceitunas es por medio de las estimaciones 01 y 02 , haciendo los cálculos y los gráficos para ver la temperatura óptima.

$$
\begin{aligned}
& \frac{\partial R E N}{\partial T M A X}=51687,07-2 *(1073,751) T M A X=0 \\
& \Rightarrow \quad T M A X=24,07
\end{aligned}
$$

Determinando el punto máximo para la temperatura máxima promedio anual.
Determinando el punto máximo para la temperatura mínima promedio anual.

$$
\begin{aligned}
& \frac{\partial R E N}{\partial T M I N}=65404,02-2 *(2014,063) T M I N=0 \\
& \Rightarrow \quad T M I N=16,24
\end{aligned}
$$

Resulta que el requerimiento óptimo de la temperatura mínima para este cultivo es $16,24^{\circ} \mathrm{C}$ y en el caso de la temperatura máxima es $24,07^{\circ} \mathrm{C}$, resultados muy aproximados con lo reportado por Tapia et al. (2003) sobre los requerimientos mínimos del clima en la producción de olivo, los cuales están entre $15{ }^{\circ} \mathrm{C}$ y $30{ }^{\circ} \mathrm{C}$. La Figura 2 muestra un comportamiento $\mathrm{U}$ invertida entre el rendimiento del cultivo y las temperaturas máximas y mínimas, indica que en las etapas iniciales de la función de producción, ante un aumento del nivel de la temperatura, se experimenta un incremento en los rendimientos del cultivo de olivo, este comportamiento persiste hasta alcanzar un punto máximo del rendimiento, a partir de este punto el rendimiento de los cultivos tienen comportamientos decrecientes.

Teniendo en consideración el promedio de las temperaturas máximas y mínimas anuales, vemos que el nivel de temperatura máxima aún no ha alcanzado a su óptimo $\left(24,07^{\circ} \mathrm{C}\right)$, lo que permite los mayores rendimientos para este cultivo, es decir, 

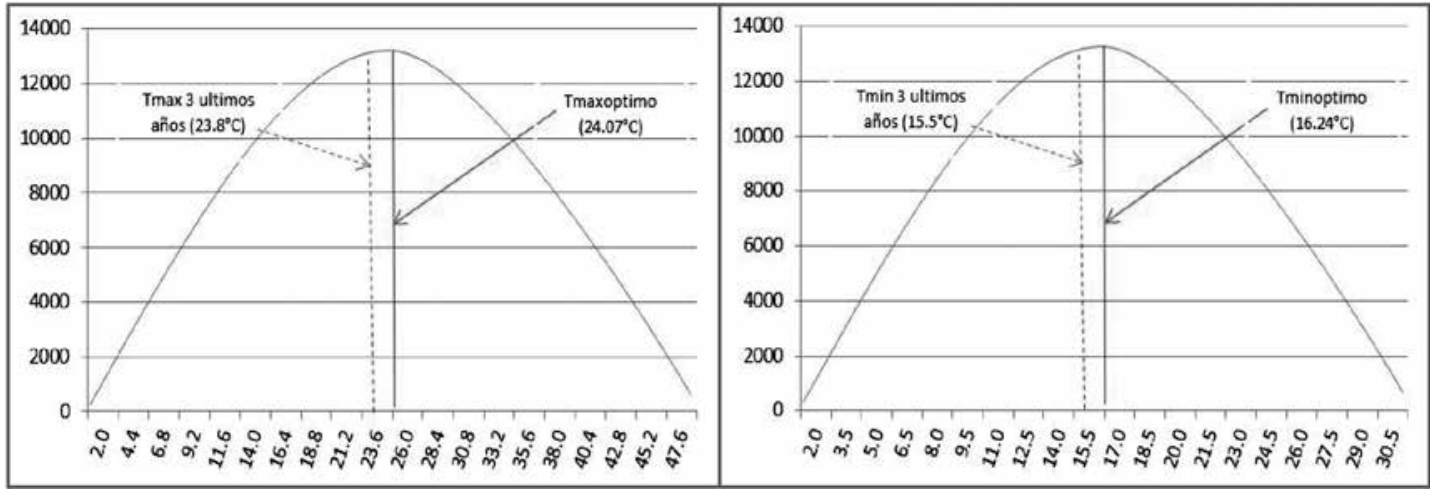

Figura 2. Temperatura Máxima y Mínima Óptima para el Olivo.

el nivel de aumentos de la temperatura benefician a la producción de aceituna; sin embargo, con el nivel de temperatura mínima estas han sobrepasado el óptimo $\left(16,24{ }^{\circ} \mathrm{C}\right)$, por lo que ya podría estar teniendo efectos desfavorables sobre este cultivo.

\section{Discusión}

El clima es un factor determinante en la productividad agrícola, por tanto es básico entender, estimar y proyectar los efectos del cambio climático en el sector agrícola, el que influye directamente en la producción de los cultivos, también en las frecuencias e intensidades de las cosechas, como sostiene Mitchell (2005). Sin embargo, esto es complejo si se considera que diferentes variables pueden interferir y que el ser humano tiende a adaptar su comportamiento ante los cambios, inclusive los efectos del cambio climático en la agricultura son diferentes entre los cultivos así como entre los países, e incluso entre regiones de un mismo país, tal como indica Seo y Mendelsohn (2007) y Galindo (2009).

Considerando la publicación de los Escenarios Climáticos en el Perú para el 2030, por la Oficina General de Estadística e Informática del SENAMHI (2010), los resultados para el escenario de emisión A2 (altas emisiones), para las zonas costeras, son como sigue: las variaciones de temperaturas máximas anuales para el 2030 crecerá en el rango de $0,8^{\circ} \mathrm{C}$ a $1,2{ }^{\circ} \mathrm{C}$; mientras, las variaciones de temperaturas mínimas anuales para el 2030 decrecerá en el rango de $0,4{ }^{\circ} \mathrm{C}$ a $0,8{ }^{\circ} \mathrm{C}$; coincidiendo con lo reportado por Cline (2007), Torres (2010), quienes en sus investigaciones indican que la variación de las temperaturas promedio extremas son de $0,2{ }^{\circ} \mathrm{C}$ por cada década.
Según los resultados de las estimaciones econométricas de la sesión anterior, se realizó la proyección basándose en los valores de rango bajo y alto de variabilidades climáticas señalados anteriormente, vemos que para el 2030 las caídas del rendimiento de la producción de aceituna en Tacna-Perú alcanzarían a 4,94\% en el nivel bajo y de $6,42 \%$ en el nivel alto, coincidiendo con Torres (2010), quien indica para el norte del Perú, los cultivos agrícolas como el mango y plátano, disminuirían sus rendimientos en $7 \%$ y $30 \%$, respectivamente. Seo y Mendelsohn (2007), para los países sudamericanos, estiman que, en promedio, productores grandes y pequeños perderán hasta el $25 \%$ del valor de su flujo de ingresos para 2060. El porcentaje se incrementa hasta $50 \%$ en el escenario climático más severo correspondiente a 2100. Ordaz et al. (2009), sostienen que el país de El Salvador sufrirá grandes pérdidas en la agricultura ante los efectos climáticos. Para el 2100 el aumento de la temperatura estará entre $2{ }^{\circ} \mathrm{C}$ y $5{ }^{\circ} \mathrm{C}$ y la precipitación aumentará entre $18 \%$ y $40 \%$, lo que traerá una reducción entre $2 \%$ y $8 \%$ del PIB comprado al PIB del 2007.

\section{Conclusiones}

Los resultados del análisis nos muestran que las variables climáticas como la temperatura máxima y mínima promedio anual, precipitación y humedad relativa del suelo, así como participación porcentual del sector agrícola en el PIB regional influyen significativamente en el rendimiento de la producción de aceituna en Yarada (Tacna), la bondad de ajuste del modelo está explicada en más del $70 \%$, el indicador de verosimilitud es alta, lo que justifica la validez y significancia del modelo considerado. 
De los resultados vemos que el cultivo de olivo tiene un umbral diferente de temperatura mínima y máxima que estarían dentro del rango de los estándares requeridos, el nivel de temperatura máxima aún no ha alcanzado su óptimo $\left(24,07^{\circ} \mathrm{C}\right)$; un aumento de temperatura promedio máxima aumenta los rendimientos para este cultivo; pasa lo contrario con el nivel de temperatura mínima, estas han sobrepasado el óptimo $\left(16,24{ }^{\circ} \mathrm{C}\right)$, por lo que las caídas de la temperatura mínima extremas podrían tener efectos desfavorables sobre este cultivo.

Considerando los Escenarios Climáticos al 2030 para zonas costeras del Perú, hecho por SENAMHI (2010), se experimenta al 2030 unos descensos leves del rendimiento de la producción de aceituna de 4,94\% en el nivel bajo y 6,42\% en el nivel alto; la caída en el rendimiento de la aceituna es independiente de si tomamos el valor bajo o alto del rango de pronóstico de clima.

\section{Literatura Citada}

Cline, W.R.

2007. Global Warming and Agriculture: Impact Estimates by Country. Center for Global Development y Peterson Institute for International Economics, Washington, D.C., pp. 178.

Fleischer, A.; Lichtman, I.; Mendelsohn R.

2007. Climate Change, Irrigation, and Israeli Agriculture: Will Warming Be Harmful? World Bank, Policy Research Working Paper, $\mathrm{N}^{\circ}$ 4135, pp. 22.

Galindo, L.M.

2009. La Economía del Cambio Climático en México, Síntesis. Facultad de Economía de la Universidad Nacional Autónoma de México, 67: 21-43.

Johansen, S.

1995. Likelihood-Based Inference in Cointegrated Vector Autoregressive Models. Oxford University Press, pp. 267.

Mitchell, J.

2005. Climate change and the greenhouse effect- A briefing from the Hadley Center-The Hadley Centre for Climate Prediction and Research, Met Office, United Kingdom, pp. 69.
Ordaz, J.; Ramírez, D.; Mora, J.

2009. El Salvador: Efectos del Cambio Climático sobre la Agricultura. Comisión Económica para América Latina y el Caribe-CEPAL, pp. 66.

Organización de las Naciones Unidas-ONU.

1992. Convención Marco de las Naciones Unidas sobre el Cambio Climático (CMNUCC), realizada en Rio de Janeiro, Brasil, pp. 50.

Servicio Nacional de Meteorología e Hidrología-SENAMHI 2010. Resumen Técnico del Cambio Climático Nacional, Lima-Perú, pp. 23.

Seo, S.N.; Mendelsohn, R.

2007. Climate Change Impacts on Animal Husbandry in Africa: A Ricardian Analysis. World Bank Policy Research Working Paper, Nº 4621. Washington, DC., pp. 48.

Tapia, F.; Ibacache, A.; Astorga, M. 2003. Manual del Cultivo de Olivo. Boletín INIA No 101. ISSN 0717-4829. La Serena Chile, 128: 11-20[.

Torres, L.

2010. Análisis Económico del Cambio Climático en la Agricultura de la Región Piura-Perú, Caso: Principales Productos Agroexportables. Consorcio de Investigación Económica y Social (CIES), Lima, Perú, pp. 101. 
